\title{
Impulse Control Disorders Following Deep Brain Stimulation of the Subthalamic Nucleus in Parkinson's Disease: Clinical Aspects
}

\author{
Polyvios Demetriades, ${ }^{1}$ Hugh Rickards, ${ }^{1}$ and Andrea Eugenio Cavanna ${ }^{1,2,3}$ \\ ${ }^{1}$ University of Birmingham Medical School, Birmingham B152TT, UK \\ ${ }^{2}$ Department of Neuropsychiatry, University of Birmingham and BSMHFT, Barberry Building, 25 Vincent Drive, \\ Birmingham B152FG, UK \\ ${ }^{3}$ Sobell Department of Motor Neuroscience and Movement Disorders, Institute of Neurology, UCL, London WC1N 3BG, UK \\ Correspondence should be addressed to Andrea Eugenio Cavanna, a.cavanna@ion.ucl.ac.uk
}

Received 14 October 2010; Accepted 7 January 2011

Academic Editor: Antonio Strafella

Copyright () 2011 Polyvios Demetriades et al. This is an open access article distributed under the Creative Commons Attribution License, which permits unrestricted use, distribution, and reproduction in any medium, provided the original work is properly cited.

\begin{abstract}
Parkinson's disease (PD) has been associated with the development of impulse control disorders (ICDs), possibly due to overstimulation of the mesolimbic system by dopaminergic medication. Preliminary reports have suggested that deep brain stimulation (DBS), a neurosurgical procedure offered to patients with treatment-resistant PD, affects ICD in a twofold way. Firstly, DBS allows a decrease in dopaminergic medication and hence causes an improvement in ICDs. Secondly, some studies have proposed that specific ICDs may develop after DBS. This paper addresses the effects of DBS on ICDs in patients with PD. A literature search identified four original studies examining a total of 182 patients for ICDs and nine case reports of 39 patients that underwent DBS and developed ICDs at some point. Data analysis from the original studies did not identify a significant difference in ICDs between patients receiving dopaminergic medication and patients on DBS, whilst the case reports showed that $56 \%$ of patients undergoing DBS had poor outcome with regards to ICDs. We discuss these ambivalent findings in the light of proposed pathogenetic mechanisms. Longitudinal, prospective studies with larger number of patients are required in order to fully understand the role of DBS on ICDs in patients with PD.
\end{abstract}

\section{Introduction}

Parkinson's disease (PD) is increasingly recognized as a neurodegenerative condition characterized by motor dysfunction and both physiological and psychological disturbances [1]. Although PD has been classically associated with psychiatric comorbidities such as dementia [2] and psychosis [3], recent studies have shown that patients with PD can develop a variety of behavioral problems associated with impulse dyscontrol, including pathological gambling, hypersexuality, punding (repetitive purposeless motor acts not distressing to the patient), and compulsive shopping and eating [4]. These pathological behaviors are currently classified as impulse control disorders (ICDs) and exert negative consequences in terms of the patients' health-related quality of life, mainly because of the interference with their social functioning [5]. The aetiopathogenesis of ICDs in patients with PD is not completely understood, but previous studies showed that dopamine replacement therapy can lead to the development of ICDs due to overstimulation of the mesolimbic dopaminergic system [6] which modulates behavioral responses to reward, motivation, and reinforcement. A recent large cross-sectional study has shown that up to $13.6 \%$ of patients with treated idiopathic PD may suffer from ICDs [7], with hypersexuality, pathological gambling, and compulsive shopping being the most common ones. Levodopa use, younger age of onset of PD, and unmarried status were associated with the development of ICDs. Other predictive factors included being male, history of alcohol abuse, and novelty seeking or impulsive personality traits [8]. Finally, it has been consistently found that patients using dopamine agonists are more likely to develop ICDs $(6.3 \%)$ than those using L-dopa (0.6\%) [9].

In some patients, dopaminergic medication becomes less effective in treating motor symptoms. Deep brain stimulation (DBS) is an effective neurosurgical procedure 
that can reduce motor symptoms in patients with treatmentrefractory PD (especially patients who developed levodopainduced dyskinesia), thus allowing decrease in their medication [10]. Consequently, DBS might have an indirect beneficial role in patients suffering from ICDs. However, DBS may also have detrimental effect on patients' behaviours. The most effective target of DBS in PD is arguably the subthalamic nucleus (STN), which plays a part in the frontostriato-thalamic-cortical loops mediating motor, cognitive, and emotional functions [11], thus suggesting that DBS may affect patients' behavior, in addition to motor performance. Both case reports and clinical studies associating DBS with the postoperative development of ICDs have provided support to this hypothesis.

As the popularity of DBS increases and this neurosurgical procedure is offered to patients suffering from other treatment-resistant movement disorders commonly associated with ICDs, such as Tourette syndrome [12], there is a need of more conclusive results on its role in the development of ICDs. This literature review assesses the current evidence on the clinical implications of ICDs in patients with PD who underwent DBS of the subthalamic nucleus.

\section{Literature Search Methodology}

We performed a literature search across the databases Medline, EMBASE, and PsycInfo to identify original studies and case reports which examined the behavioral effects of DBS in patients with PD, with focus on ICDs, as defined in Chapter 5 of the ICD-10 classification system [5]. We used the search terms "Parkinson," "deep brain stimulation", "impulse control disorder", "impulsivity", "hypersexuality", "pathological gambling", "punding", "compulsive shopping", "compulsive eating", and "addiction", and we limited our search to papers published in English language.

\section{Original Studies}

Four original studies met our search criteria, examining a total of 182 patients for ICDs. We selectively reviewed studies reporting patients with ICDs. As a result, due to publication bias, the prevalence of ICDs after DBS cannot be extrapolated from the reviewed data.

Two studies (Funkiewiez et al. [13] and Contarino et al. [14]) followed up patients with PD after DBS surgery, in order to identify any changes in their behavioral profile, including impulse dyscontrol. The other two studies (Hälbig et al. [15] and Czernecki et al. [16]) performed a crosssectional evaluation comparing patients with PD-treated DBS (PD + DBS) with those treated with dopaminergic medication (levodopa and/or dopamine agonists) (PD + DA). A proportion of patients from the latter group remained on medication postoperatively.

Table 1 summarizes the demographic and clinical characteristics of the two groups of patients (PD + DBS and PD $+\mathrm{DA}$ ) across the four studies. The PD + DBS group was larger than the PD + DA group since only two of the four studies included controls ( $P D+D A)$. The mean age of the
TABLE 1: Summary of demographic and clinical characteristics of patients with Parkinson's disease from four original studies evaluating the effects of deep brain stimulation on impulse control disorders.

\begin{tabular}{lcc}
\hline & PD + DBS & PD + DA \\
\hline No. of patients & 122 & 60 \\
Gender: M/F/unknown & $62 / 42 / 18$ & $26 / 11 / 23$ \\
Mean age (years) & 55 & 57 \\
Mean disease duration (years) & 14 & 10 \\
\hline
\end{tabular}

Abbreviations. PD: Parkinson's disease; PD + DBS: patients with Parkinson's disease who underwent deep brain stimulation surgery (some remained on medication postoperatively); PD + DA: patients with Parkinson's disease treated with dopaminergic medication.

patients was similar in both groups whereas disease duration was higher in PD + DBS than in PD + DA, consistent with the clinical practice of referring to neurosurgery patients with longer disease duration who failed to respond to medication. Motor evaluation of the patients in all studies was performed using the Unified Parkinson's Disease Rating Scale III (UPDRS III). In the PD + DBS group, the UPDRS III ranged from 10.1 ( $\mathrm{SD} \pm 2.4)$ (Czernecki et al.) to 20.8 $(\mathrm{SD} \pm 12.1)$ (Contarino et al.), whereas in the $\mathrm{PD}+\mathrm{DA}$ group, the UPDRS III scores ranged from 16.3 (SD \pm 10.6 ) (Hälbig et al.) to 38.7 ( $\mathrm{SD} \pm 2.8$ ) (Czernecki et al.). Exclusion criteria included severe psychiatric conditions and cognitive impairment measured by Minimental State Examination (MMSE), possibly masking severe cases of ICDs.

Unfortunately, the methodology of each study differed, and hence no conclusive results can be drawn from the patients as a group. Table 2 outlines the total number of patients in each group $(\mathrm{PD}+\mathrm{DBS}$ and $\mathrm{PD}+\mathrm{DA})$ and the percentage of each group who developed ICDs.

Hälbig et al. [15] identified a higher percentage of patients with newly developed ICDs in the PD + DBS group $(19 \%)$ than in the PD + DA group (8\%), but this was not statistically significant with $P=.26$ (Table 2 ). However, this study identified a statistically significant difference in overall impulsivity $(P=.04)$ between the two groups, with $\mathrm{PD}+$ DBS reporting higher impulsivity scores (Table 3 ). Notably, preoperative assessment of ICDs was not performed, so the two groups may had been different from the start. All of the patients in the PD + DBS group were with stimulation "on" at the time of the assessment and remained on dopaminergic medication after surgery (56\% on levodopa monotherapy, $44 \%$ on combined levodopa and dopamine agonist therapy) with mean LEDD 682mg ( $\mathrm{SD} \pm 427 \mathrm{mg}$ ). The $\mathrm{PD}+\mathrm{DA}$ group's mean LEDD was $582 \mathrm{mg}$ (SD $\pm 480 \mathrm{mg}$ ), with $38 \%$ of the patients on levodopa monotherapy, $16 \%$ on dopamine agonist monotherapy, and $46 \%$ on combined therapy.

Contarino et al. [14] and Funkiewiez et al. [13] reported the development of ICDs in 18\% (hypersexuality) and $2.5 \%$ (aggressive impulsive behaviour) of their patients (Table 2). Czernecki et al. [16] used methods described in Rolls et al. [17] and Bechara et al. [18] to compare the reward sensitivity (an indirect measure of impulsivity) of patients receiving DBS (in both "on" and "off" conditions) with patients 
TABle 2: Development of impulse control disorders after deep brain stimulation or dopaminergic pharmacotherapy in patients with Parkinson's disease.

\begin{tabular}{|c|c|c|c|c|c|}
\hline \multirow{2}{*}{ Study } & \multirow{2}{*}{ Type of study } & \multicolumn{2}{|c|}{ Number of patients in each study; $\mathrm{n}$} & \multicolumn{2}{|c|}{ Number of patients developing ICDs; $\mathrm{n}(\%)$} \\
\hline & & $\mathrm{PD}+\mathrm{DBS}$ & $\mathrm{PD}+\mathrm{DA}$ & $\mathrm{PD}+\mathrm{DBS}$ & $\mathrm{PD}+\mathrm{DA}$ \\
\hline Halbig et al. & Cross-sectional & 16 & 37 & $3(19 \%)$ & $3(8 \%)$ \\
\hline Contarino et al. & Longitudinal & 11 & 0 & $2(18 \%)$ & $\mathrm{n} / \mathrm{a}$ \\
\hline Funkiewiez et al. & Longitudinal & 77 & 0 & $2(2.5 \%)$ & $\mathrm{n} / \mathrm{a}$ \\
\hline Czernecki et al. & Cross-sectional & 18 & 23 & $\mathrm{n} / \mathrm{a}$ & $\mathrm{n} / \mathrm{a}$ \\
\hline
\end{tabular}

Abbreviations. ICDs: impulse control disorders; PD: Parkinson's disease; PD + DBS: patients with Parkinson's disease who underwent deep brain stimulation surgery; PD + DA: patients with Parkinson's disease treated with dopaminergic medication (levodopa and dopamine agonists).

TABLE 3: Measures of impulsivity after deep brain stimulation or dopaminergic pharmacotherapy in patients with Parkinson's disease.

\begin{tabular}{|c|c|c|c|c|c|}
\hline Study & \multicolumn{2}{|c|}{ Outcome measures } & $\mathrm{PD}+\mathrm{DBS}$ & $\mathrm{PD}+\mathrm{DA}$ & $P$ value \\
\hline Halbig et al. & $\begin{array}{l}\text { Barratt impulsiveness } \\
\text { Scale; mean }(\mathrm{SD})\end{array}$ & $\begin{array}{c}\text { "on" } \\
\text { treatment }\end{array}$ & $44.97(17.29)$ & $36.11(17.29)$ & 0.04 \\
\hline \multirow{8}{*}{ Czernecki et al. } & \multirow{2}{*}{$\begin{array}{l}\text { Stimulus reward } \\
\text { association learning } \\
\text { (number of trials)*; } \\
\text { mean (SEM) }\end{array}$} & $\begin{array}{l}\text { "on" } \\
\text { treatment }\end{array}$ & $19.2(3.9)$ & $22.6(5.6)$ & \multirow[t]{2}{*}{0.37 (group) $^{* *}$} \\
\hline & & $\begin{array}{l}\text { "off" } \\
\text { treatment }\end{array}$ & $23.1(4.6)$ & $28.8(4.8)$ & \\
\hline & \multirow{2}{*}{$\begin{array}{l}\text { Reversal* (number in } \\
30 \text { trials); mean } \\
\text { (SEM) }\end{array}$} & $\begin{array}{l}\text { "on" } \\
\text { treatment }\end{array}$ & $1.6(0.2)$ & $1.3(0.2)$ & \multirow[t]{2}{*}{0.48 (group) $)^{* *}$} \\
\hline & & $\begin{array}{c}\text { "off" } \\
\text { treatment }\end{array}$ & $1.3(0.2)$ & $1.3(0.2)$ & \\
\hline & \multirow[t]{2}{*}{$\begin{array}{l}\text { Extinction* (last } \\
\text { error); mean (SEM) }\end{array}$} & $\begin{array}{l}\text { "on" } \\
\text { treatment }\end{array}$ & $8.1(1.1)$ & $14.2(2.5)$ & \multirow[t]{2}{*}{0.13 (group) $^{* *}$} \\
\hline & & $\begin{array}{l}\text { "off" } \\
\text { treatment }\end{array}$ & $10.5(1.6)$ & $11.8(1.9)$ & \\
\hline & \multirow[t]{2}{*}{$\begin{array}{l}\text { Gambling task; mean } \\
(\mathrm{SEM})\end{array}$} & $\begin{array}{l}\text { "on" } \\
\text { treatment }\end{array}$ & $25.4(10.2)$ & $13.4(6.9)$ & \multirow[t]{2}{*}{$0.39{\text { (group })^{* *}}^{*}$} \\
\hline & & $\begin{array}{c}\text { "off" } \\
\text { treatment }\end{array}$ & $19.4(9.6)$ & $14.2(6.4)$ & \\
\hline
\end{tabular}

In all measures (except the Barratt impulsiveness scale and the extinction test), lower scores indicate higher impulsivity.

* Tasks described in Rolls et al. [17] **ANOVA results of $P$-value of group effect (patients on stimulation versus patients on medication)

Abbreviations. PD + DBS: patients with Parkinson's disease who underwent deep brain stimulation surgery; PD + DA: patients with Parkinson's disease treated with dopaminergic medications (levodopa and/or dopamine agonists); "on" treatment: on medication and stimulation (if applicable) on the time of assessment; "off" treatment: off medication and stimulation.

receiving dopaminergic medication. The methods included stimulus reward learning with either reversal or extinction and the gambling task (Table 3 ). In the stimulus reward learning procedure, the subjects were faced with a screen challenging them to learn how to choose the correct pattern in order to gain maximum points and to progress to further trials. The task continued to either the reversal phase (testing sensitivity to reward flexibility) or the extinction phase (testing the ability to control impulsivity). The gambling task included making a choice between advantageous (small gains but smaller loses) and disadvantageous (big gains but bigger loses) decks. Previous reports by the same authors using the same tasks identified that patients with PD had impaired explicit and implicit reinforcement-associated learning and "sensitivity to reward" flexibility, compared to healthy controls [19]. In this study, there was no difference between the PD + DBS and PD + DA groups, implying that although patients with PD may have higher impulsivity than controls, DBS does not seem to increase it further. In this study, the preoperative LEDD of the PD + DBS group did not differ significantly from the PD + DA group, whilst the postoperative mean LEDD of the PD + DBS group was $133.3 \mathrm{mg}(\mathrm{SEM} \pm 58.7)$, compared to $982.3 \mathrm{mg}(\mathrm{SEM} \pm 53.9)$ in the $\mathrm{PD}+\mathrm{DA}$ group.

\section{Case Reports}

We found 9 case reports of 39 patients with PD (33 men and 6 women) who underwent DBS and developed ICDs or whose ICDs worsened $(n=22)$. In other cases $(n=$ 17), patients presented with ICDs that resolved or improved postoperatively. Although our study selection again involves publication bias, the case reports will provide detailed information about the phenomenology of ICDs and the 
clinical characteristics of the patients who develop ICDs. The demographic and clinical characteristics for each patient are described in Table 4.

Romito et al. [20], Doshi and Bhargava [21], Smeding et al. [22], Sensi et al. [23], and Lim et al. [24] reported patients with no preoperative ICDs who developed ICDs after surgery (patients $1-7,19,38$ ) (Table 5). The time period between surgery and the development of ICD varied in each case from immediately after surgery to months later. Most of the ICDs were transient and resolved within a year of their development. Lim et al. [24] described a series of patients with preoperative ICDs that worsened after surgery (patients 20-32); some of these patients developed a wider spectrum of ICDs after the operation compared to what they had preoperatively (patients 20-22, 31-32).

Witjas et al. [25], Bandini et al. [26], Ardouin et al. [27], and Lim et al. [24] described patients with preoperative ICDs that resolved completely (patients 8-18, 33-35, 39) or improved significantly (patients $36-37$ ) postoperatively. In all cases, there was a significant reduction (reduction to less than $50 \%$ of preoperative dose) in levodopa equivalent daily dose (LEDD), most commonly in the first 3 months after DBS. Most of the studies provided the LEDD for the patients; where not explicitly stated, it was calculated as described in Wenzelburger et al. [28].

Analysis of the findings of the case reports revealed that 22 patients $(1-7,19-32,38)$ had a "poor outcome" after DBS, defined as worsening of existing ICDs, new development of ICDs, or no postoperative improvement of existing ICDs, and 17 patients $(8-18,33-37,39)$ had a "good outcome" after DBS, defined as improvement of existing ICDs or resolution of existing ICDs postoperatively (Table 5). Of the patients with "poor outcome", 91.1\% were males and $72.7 \%$ were older than 55 years, whereas in the "good outcome" group, only $76.5 \%$ were males and $70 \%$ of them were older than 55 years. Age information is not available for all patients in the "good outcome" group. Previous psychiatric history and postoperative psychiatric conditions were not available for all the patients. However, it was noted that only $36.4 \%$ of patients in the "good outcome" group had previous behavioral problems, compared to $42.9 \%$ in the "poor outcome" group. The patients who developed ICDs or whose ICDs worsened after DBS had a $36.4 \%$ chance of developing other psychiatric conditions. These included depression, manic syndrome, agitation, anxiety, and psychosis. On the other hand, only $17.7 \%$ of the patients in the "good outcome" group developed other psychiatric conditions postoperatively (depression).

\section{Comments and Clinical Implications}

This review paper specifically examined the literature on the role of DBS of the subthalamic nucleus on ICDs in patients with $\mathrm{PD}$. In general, the existing evidence on this topic is poor, not allowing definitive conclusions whether DBS benefits patients with ICDs or whether it actually causes the development of ICDs.
5.1. Development of ICDs after DBS. Three out of the four studies described in Tables 1-3 reported the development of ICDs after DBS surgery. These findings are in agreement with 22 case reports of patients who had a poor postoperative outcome in terms of ICDs. In most cases, the ICDs developed despite a postsurgical reduction of dopaminergic medication. The reasons for the development of ICDs after DBS are still unknown, and hence a lot of different hypothesis have been proposed.

The first hypothesis suggests that STN-DBS increases impulsivity by stimulating the STN or neighboring fiber tracts, since the STN is interconnected with structures linked to both associative and limbic circuits within the basal ganglia. It is proposed that the STN can regulate the processing of associative and limbic information towards cortical and subcortical regions, thus influencing changes in behavior [29]. This is compatible with studies suggesting that the STN mediates not only motor but also cognitive and emotional functions [30]. Even the surgical procedure itself may have an adverse result on impulsivity, since the STN is a very compact structure, and it may be difficult to selectively influence the motor part of it without damaging it or its neighboring structures which are associated with motivational behaviors. This hypothesis was proposed by Sensi et al., who suggested that a microtraumatic effect of surgery or a misplacement of the electrodes may be the reason behind the development of ICDs after DBS [23]. Moreover, minimal changes in the stimulation site can cause different effects; Smeding et al. [22] showed that with the stimulation of the most dorsal contact of the STN nucleus, the performance on decision making tasks is worse than with stimulation of the ventral contact.

Previous reports have shown that in medically treated patients, higher levodopa dosages are associated with the development of ICDs [7]. Hence, a possible explanation for the development of ICDs after DBS is the higher cumulative exposure to dopaminergic medication due to longer disease duration and greater disease severity of the patients who underwent DBS. However, this hypothesis would not explain why some patients developed ICDs ex novo after surgery. Further complicating the picture, postoperative dopaminergic therapy reduction is also likely to play an important role. As seen in the case reports, unlike $100 \%$ of patients in the "good outcome" group, only $57.1 \%$ of patients in the "poor outcome" group had a significant reduction in their medication. This means that if preoperative ICDs exist, only a significant reduction in the patient's medication would improve the patient's impulsivity [26]. Moreover, the reduction would presumably be allowed only if the patient's motor symptoms improve after surgery [25]. This explanation may also be supported by the findings of the study by Halbig et al., where surgery did not allow a major reduction in medication postoperatively (with $\mathrm{PD}$ + DBS and PD + DA having similar LEDD). Hence, in the probable background of preoperative ICDs and with a high dose of postoperative dopaminergic medication, the authors identified higher impulsivity in the PD + DBS group. Patients who remained on dopaminergic medication postoperatively had a poor outcome in terms of ICDs possibly because DBS 


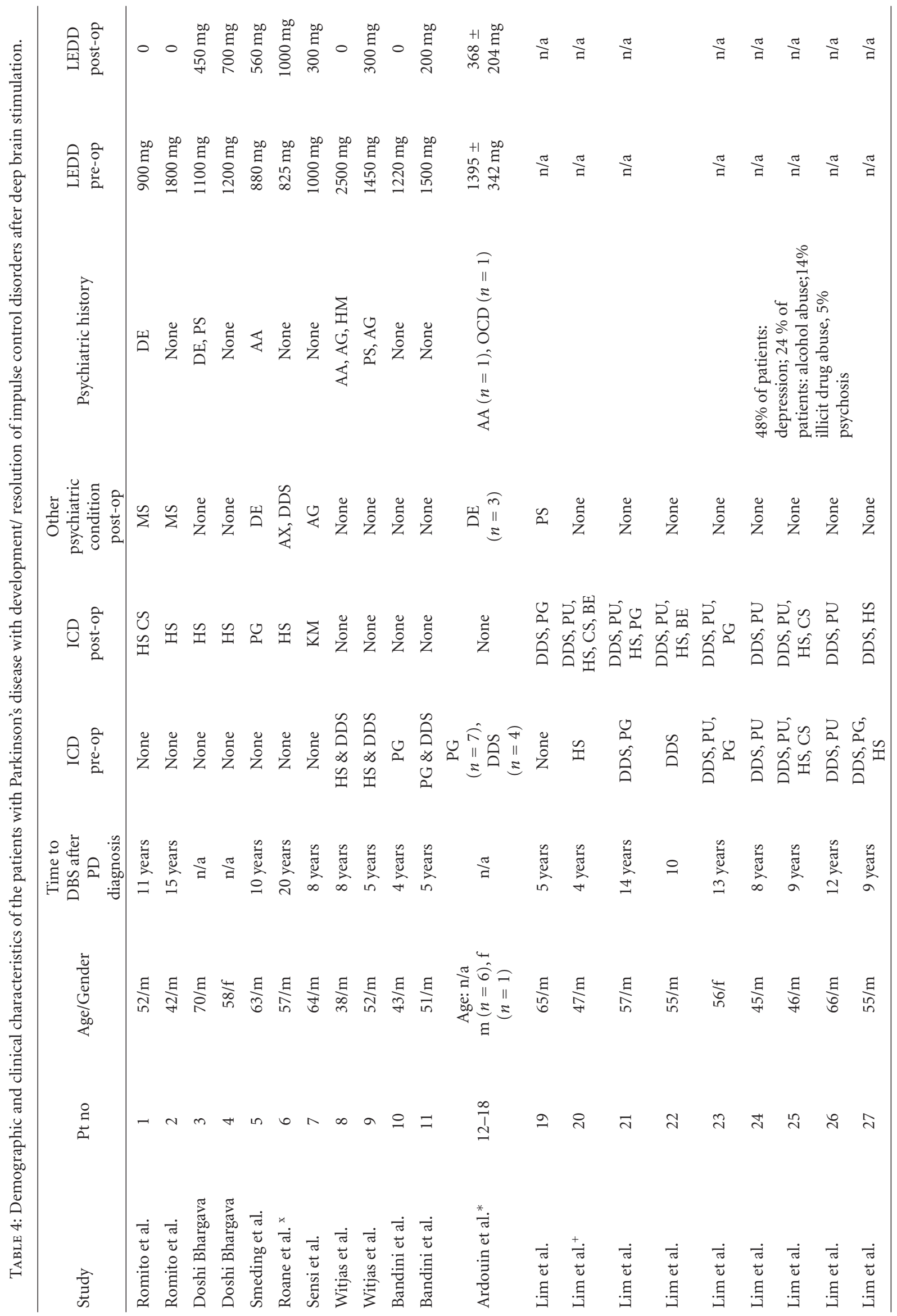




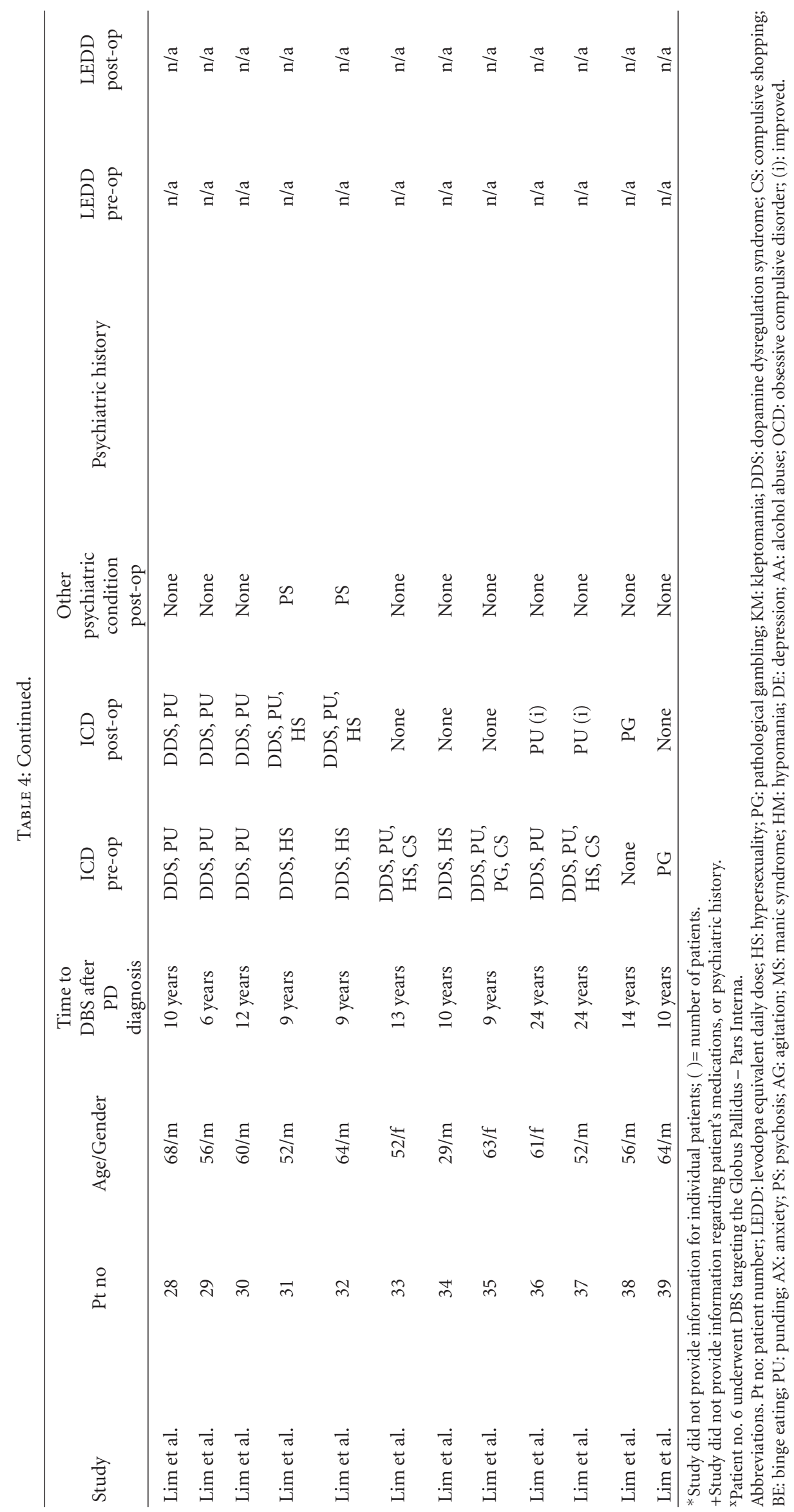


TABLE 5: Analysis of the outcomes in the case reports presented in Table 4.

\begin{tabular}{lcc}
\hline & Poor outcome & Good outcome \\
\hline Total Number & 22 & 17 \\
Male gender $(\%)$ & $20 / 22(91.1)$ & $13 / 17(76.5)$ \\
Age $\geq 55$ years & $16 / 22(72.7)$ & $7 / 10^{*}(70)$ \\
Post-op medication reduction to less than $50 \%$ of pre-op dose & $4 / 7^{*}(57.1)$ & $11 / 11^{*}(100)$ \\
Psychiatric history & $3 / 7^{*}(42.9)$ & $4 / 11^{*}(36.4)$ \\
Other psychiatric condition post-op & $8 / 22(36.4)$ & $3 / 17(17.7)$ \\
\hline
\end{tabular}

"Poor outcome" is defined as worsening of existing ICDs, development of more ICDs, or no improvement of existing ICDs postoperatively; "good outcome" is defined as improvement of existing ICDs, disappearance of existing ICDs postoperatively.

* Information not available for the rest of the patients.

can sensitize the brain to the behavioral effects of dopamine replacement therapy, especially on the background of high impulsivity [22].

Finally, a less likely explanation for the appearance of ICDs after DBS surgery is that the improvement of motor function in some patients may have facilitated the full expression of behavioral abnormalities that had been developed by the use of dopamine replacement therapy before the operation, as suggested by Houeto et al. [31].

5.2. Improvement of ICDs after DBS. A number of case reports in this paper described patients whose ICDs either resolved or markedly improved after surgery. However, the potential role of DBS in the resolution of ICDs is far from being clear.

Different mechanisms have been proposed to explain the resolution of ICDs after DBS. First of all, it is well known that dopaminergic stimulation is implicated in impulsive behaviors. Moreover, it has been consistently shown that dopaminergic medications, and in particular dopamine agonists, may predispose patients with PD to the development of ICDs [32]. The significant motor improvement after DBS allows an important reduction in dopamine replacement therapy and thus a less pulsatile and nonsuprathreshold stimulation of the mesolimbic dopamine receptors [27]. Table 4 reveals that all the patients in the "good outcome" group had a significant reduction in their medication. Additionally, Czernecki et al. did not identify higher reward sensitivity in the $\mathrm{PD}$ + DBS group compared to controls, probably because the postoperative mean LEDD in the former group was much lower than in the latter. Moreover, Lim et al. [24] stated that the ICDs persisted or even worsened in patients who had to remain on high dopamine replacement therapy due to poor or moderate motor benefit after DBS. Consistent to this hypothesis, Ardouin et al. [27] observed that the progressive improvement of the symptoms of their patient closely matched the reduction in his medication.

Another possible explanation is that the improvement of ICDs was due to a direct effect of DBS on the reward seeking brain circuitry [33]. The inhibition of neuronal circuits resulting from STN-DBS may affect the direct ascending dopaminergic and serotoninergic pathways towards the limbic area which are known to have a role in positive reinforcing behaviors [25]. Further studies with long-term followup of patients after DBS using impulsivity measures and neuroimaging techniques may provide useful information on the role of DBS in resolution of ICDs.

5.3. Outcome Predictors. Our results agree with previous studies suggesting that male sex, younger age of PD onset, and previous psychiatric history are predisposing factors to the development of ICDs during the disease course $[7,8]$.

Most of the patients in the reviewed studies were of male gender, in agreement with the existing literature that females are less likely to develop ICDs. However, other reasons may also explain this; patients with ICDs rarely volunteer and often deny having these behaviors. Being a female may exacerbate this effect since most of these conditions (hypersexuality, compulsive eating, and pathological gambling) are perceived as socially inappropriate and stigmatizing. It was also noted that being female resulted in better outcome after surgery; however, the role of confounding factors like age of disease onset and medication dose should be taken into account.

Although studies suggested that younger patients may be more compliant to medication changes and therefore are less likely to develop ICDs postoperatively [25], the analysis of the case reports revealed that the number of patients over 55 in the "good outcome" and "poor outcome" groups were similar.

A number of patients in the "poor outcome" group were diagnosed with PD early in their life. This is consistent with previous studies showing that patients with young onset $\mathrm{PD}$ are more likely to present with ICDs [34], findings which may reflect a confounding effect of prescribing of dopamine agonists to younger PD patients [35].

Only a few types of ICDs were reported in this paper. Hypersexuality and pathological gambling are the most commonly reported and are clearly disabling not only to the patient but also to his/her relatives. Sometimes, the family of the patient is more likely to volunteer this information to the psychiatrist. This is probably the reason why less distressing ICDs (e.g., compulsive shopping) were reported less commonly. It is important for the doctor to be aware of the possibility of development of ICDs in PD patients in order to provide support to the patient as quickly as possible.

Although information regarding the presence of previous psychiatric disorders was not always available, more patients 
in the "poor outcome" group had history of psychiatric disorders compared to those in the "good outcome." This suggests a possible association between previous psychiatric disorders/ history of abuse and ICDs.

Finally, fewer patients in the "good outcome" group suffered from psychiatric comorbidities after surgery compared to those in the "poor outcome" group. This was expected as the most common psychiatric disorder was depression, which would most likely be related to the poor outcome of the patient as the disease progresses. This stresses the importance of postoperative followup of these patients in order to treat any other developing conditions.

5.4. Limitations. At present, there is a clear need for more conclusive results on the effects of DBS on impulsivity in patients with PD. The small number of patients in the original studies did not allow to reach statistical significance and two studies lacked a control group (PD + DA). Additionally, the studies had different follow-up periods in which ICDs may had been missed. Although similar eligibility criteria may have made the patients comparable, some of the exclusion criteria, including cognitive impairment, may have introduced a bias, since patients with ICDs are more likely to develop psychiatric comorbidities. Moreover, patients were lost to follow-up and most likely these patients had worse outcomes than the rest, further biasing the sample. The authors adopted different methodologies for the analysis of their results, thus making direct comparison impossible. Some studies had poor information regarding the patients' condition before DBS surgery, suggesting that some groups may had been different from the start. Finally, the patients who had ICDs at the baseline which was not recorded could have been more likely to choose to undergo DBS, thus introducing selection bias. To draw more accurate results, controlled longitudinal studies with baseline impulsivity measures and long-term follow-up should be conducted on large numbers of subjects undergoing DBS.

\section{Conclusion}

The results of this literature review show that existing data are ambivalent about the role of DBS on impulsivity in patients with PD. Several studies support the idea that DBS is an effective indirect treatment of ICDs, whilst other studies report the worsening or ex novo development of ICDs after surgery. Various pathophysiological mechanisms, including altered dopaminergic stimulation, have been proposed to explain both situations. The selection of patients for DBS should be extremely careful in order to avoid surgery on patients who are already predisposed to develop ICDs. Finally, since the patients are not likely to volunteer their symptoms, it is important to recognize ICDs both preoperatively and postoperatively, and to adopt a multidisciplinary team approach to diagnose and manage the patients accordingly.

\section{References}

[1] P. Kumar and M. Clark, Kumar and Clark's: Clinical Medicine, WB Saunders, London, UK, 2009.
[2] M. J. Docherty and D. J. Burn, "Parkinson's Disease Dementia," Current Neurology and Neuroscience Reports, vol. 10, no. 4, pp. 292-298, 2010.

[3] W. Poewe, "Psychosis in Parkinson's disease," Movement Disorders, vol. 18, no. 6, pp. S80-S87, 2003.

[4] P. R. Burkhard, S. Catalano-Chiuvé, A. Gronchi-Perrin, A. Berney, F. J. G. Vingerhoets, and C. Lüscher, "Impulse control disorders and Parkinson's diseaseTroubles du contrôle des impulsions et maladie de Parkinson," Revue Medicale Suisse, vol. 4, no. 156, pp. 1145-1150, 2008.

[5] World Health Organisation, "International Statistical Classification of Diseases and Related Health Problems: Chapter 5 Mental and Behavioural disorders," 2007, http://www .who.int/classifications/apps/icd/icd10online.

[6] D. Grosset, "Dopamine agonists and therapy compliance," Neurological Sciences, vol. 29, no. 5, pp. S375-S376, 2008.

[7] D. Weintraub, J. Koester, M. N. Potenza et al., "Impulse control disorders in Parkinson disease: a cross-sectional study of 3090 patients," Archives of Neurology, vol. 67, no. 5, pp. 589-595, 2010.

[8] K. I. Fujimoto, "Pathological gambling and Parkinson disease," Brain and Nerve, vol. 60, no. 9, pp. 1039-1046, 2008.

[9] W. Fan, H. Ding, J. Ma, and P. Chan, "Impulse control disorders in Parkinson's disease in a Chinese population," Neuroscience Letters, vol. 465, no. 1, pp. 6-9, 2009.

[10] E. Moro, M. Scerrati, L. M. A. Romito, R. Roselli, P. Tonali, and A. Albanese, "Chronic subthalamic nucleus stimulation reduces medication requirements in Parkinson's disease," Neurology, vol. 53, no. 1, pp. 85-90, 1999.

[11] A. Parent and L. N. Hazrati, "Functional anatomy of the basal ganglia. II. The place of subthalamic nucleus and external pallidum in basal ganglia circuitry," Brain Research Reviews, vol. 20, no. 1, pp. 128-154, 1995.

[12] M. Porta, A. Brambilla, A. E. Cavanna et al., "Thalamic deep brain stimulation for treatment-refractory Tourette syndrome: two-year outcome," Neurology, vol. 73, no. 17, pp. 1375-1380, 2009.

[13] A. Funkiewiez, C. Ardouin, E. Caputo et al., "Long term of bilateral subthalamic nucleus stimulation on cognitive function, mood, and behaviour in Parkinson's disease," Journal of Neurology, Neurosurgery and Psychiatry, vol. 75, no. 6, pp. 834839, 2004.

[14] M. F. Contarino, A. Daniele, A. H. Sibilia et al., "Cognitive outcome 5 years after bilateral chronic stimulation of subthalamic nucleus in patients with Parkinson's disease," Journal of Neurology, Neurosurgery and Psychiatry, vol. 78, no. 3, pp. 248252, 2007.

[15] T. D. Hälbig, W. Tse, P. G. Frisina et al., "Subthalamic deep brain stimulation and impulse control in Parkinson's disease," European Journal of Neurology, vol. 16, no. 4, pp. 493-497, 2009.

[16] V. Czernecki, B. Pillon, J. L. Houeto et al., "Does bilateral stimulation of the subthalamic nucleus aggravate apathy in Parkinson's disease?" Journal of Neurology, Neurosurgery and Psychiatry, vol. 76, no. 6, pp. 775-779, 2005.

[17] E. T. Rolls, J. Hornak, D. Wade, and J. McGrath, "Emotionrelated learning in patients with social and emotional changes associated with frontal lobe damage," Journal of Neurology Neurosurgery and Psychiatry, vol. 57, no. 12, pp. 1518-1524, 1994.

[18] A. Bechara, H. Damasio, and A. R. Damasio, "Emotion, decision making and the orbitofrontal cortex," Cerebral Cortex, vol. 10, no. 3, pp. 295-307, 2000. 
[19] V. Czernecki, B. Pillon, J. L. Houeto, J. B. Pochon, R. Levy, and B. Dubois, "Motivation, reward, and Parkinson's disease: influence of dopatherapy," Neuropsychologia, vol. 40, no. 13, pp. 2257-2267, 2002.

[20] L. M. Romito, M. Raja, A. Daniele et al., "Transient mania with hypersexuality after surgery for high-frequency stimulation of the subthalamic nucleus in Parkinson's disease," Movement Disorders, vol. 17, no. 6, pp. 1371-1374, 2002.

[21] P. Doshi and P. Bhargava, "Hypersexuality following subthalamic nucleus stimulation for Parkinson's disease," Neurology India, vol. 56, no. 4, pp. 474-476, 2008.

[22] H. M. M. Smeding, A. E. Goudriaan, E. M. J. Foncke, P. R. Schuurman, J. D. Speelman, and B. Schmand, "Pathological gambling after bilateral subthalamic nucleus stimulation in Parkinson disease," Journal of Neurology, Neurosurgery and Psychiatry, vol. 78, no. 5, pp. 517-519, 2007.

[23] M. Sensi, R. Eleopra, M. A. Cavallo et al., "Explosiveaggressive behavior related to bilateral subthalamic stimulation," Parkinsonism and Related Disorders, vol. 10, no. 4, pp. 247-251, 2004.

[24] S.-Y. Lim, S. S. O’Sullivan, K. Kotschet et al., "Dopamine dysregulation syndrome, impulse control disorders and punding after deep brain stimulation surgery for Parkinson's disease," Journal of Clinical Neuroscience, vol. 16, no. 9, pp. 1148-1152, 2009.

[25] T. Witjas, C. Baunez, J. M. Henry et al., "Addiction in Parkinson's disease: impact of subthalamic nucleus deep brain stimulation," Movement Disorders, vol. 20, no. 8, pp. 10521055, 2005.

[26] F. Bandini, A. Primavera, M. Pizzorno, and L. Cocito, "Using STN DBS and medication reduction as a strategy to treat pathological gambling in Parkinson's disease," Parkinsonism and Related Disorders, vol. 13, no. 6, pp. 369-371, 2007.

[27] C. Ardouin, V. Voon, Y. Worbe et al., "Pathological gambling in Parkinson's disease improves on chronic subthalamic nucleus stimulation," Movement Disorders, vol. 21, no. 11, pp. 1941-1946, 2006.

[28] R. Wenzelburger, B. R. Zhang, S. Pohle et al., "Force overflow and levodopa-induced dyskinesias in Parkinson's disease," Brain, vol. 125, no. 4, pp. 871-879, 2002.

[29] Y. Temel, A. Kessels, S. Tan, A. Topdag, P. Boon, and V. VisserVandewalle, "Behavioural changes after bilateral subthalamic stimulation in advanced Parkinson disease: a systematic review," Parkinsonism and Related Disorders, vol. 12, no. 5, pp. 265-272, 2006.

[30] L. Mallet, M. Schüpbach, K. N'Diaye et al., "Stimulation of subterritories of the subthalamic nucleus reveals its role in the integration of the emotional and motor aspects of behavior," Proceedings of the National Academy of Sciences of the United States of America, vol. 104, no. 25, pp. 10661-10666, 2007.

[31] J. L. Houeto, V. Mesnage, L. Mallet et al., "Behavioural disorders, Parkinson's disease and subthalamic stimulation," Journal of Neurology Neurosurgery and Psychiatry, vol. 72, no. 6, pp. 701-707, 2002.

[32] D. Weintraub and M. N. Potenza, "Impulse control disorders in Parkinson's disease," Current Neurology and Neuroscience Reports, vol. 6, no. 4, pp. 302-306, 2006.

[33] L. Mallet, V. Mesnage, J. L. Houeto et al., "Compulsions, Parkinson's disease, and stimulation," Lancet, vol. 360, no. 9342, pp. 1302-1304, 2002.

[34] D. Weintraub, A. D. Siderowf, M. N. Potenza et al., "Association of dopamine agonist use with impulse control disorders in Parkinson disease," Archives of Neurology, vol. 63, no. 7, pp. 969-973, 2006.
[35] V. Voon, T. Thomsen, J. M. Miyasaki et al., "Factors associated with dopaminergic drug-related pathological gambling in Parkinson disease," Archives of Neurology, vol. 64, no. 2, pp. 212-216, 2007. 


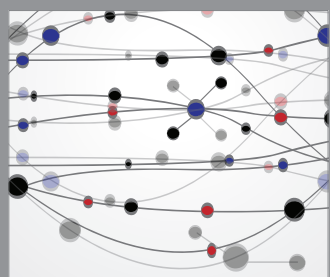

The Scientific World Journal
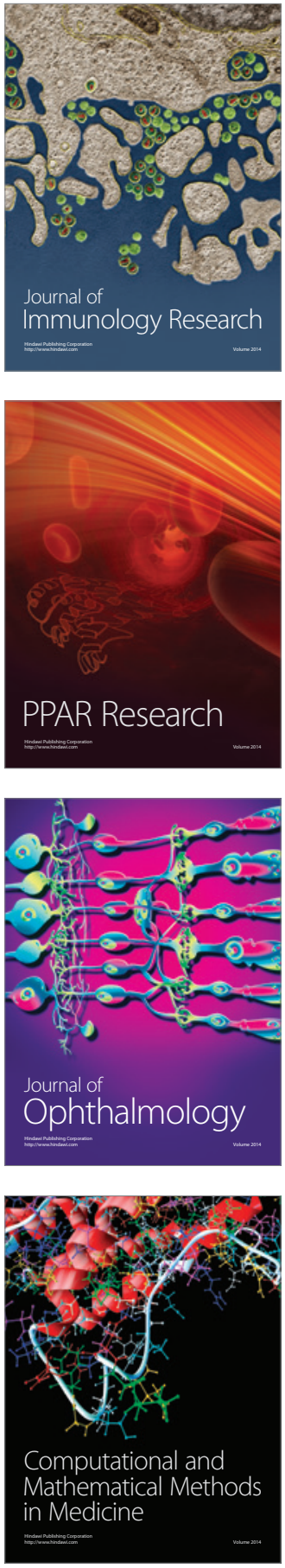

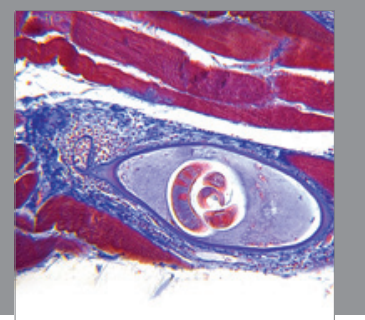

Gastroenterology

Research and Practice
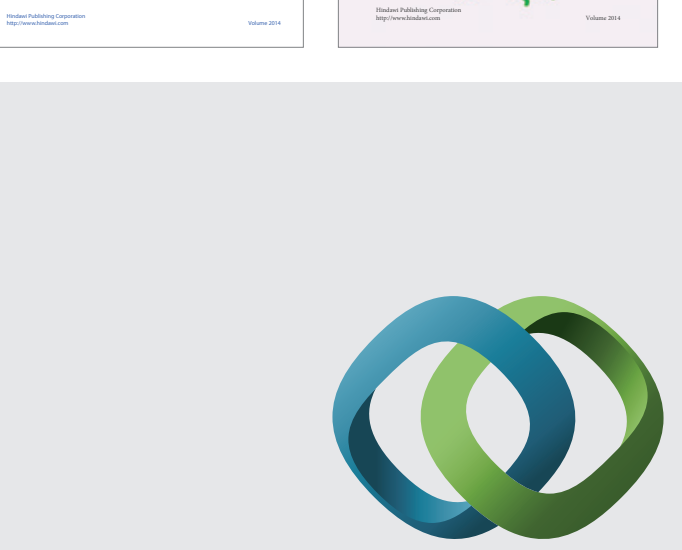

\section{Hindawi}

Submit your manuscripts at

http://www.hindawi.com
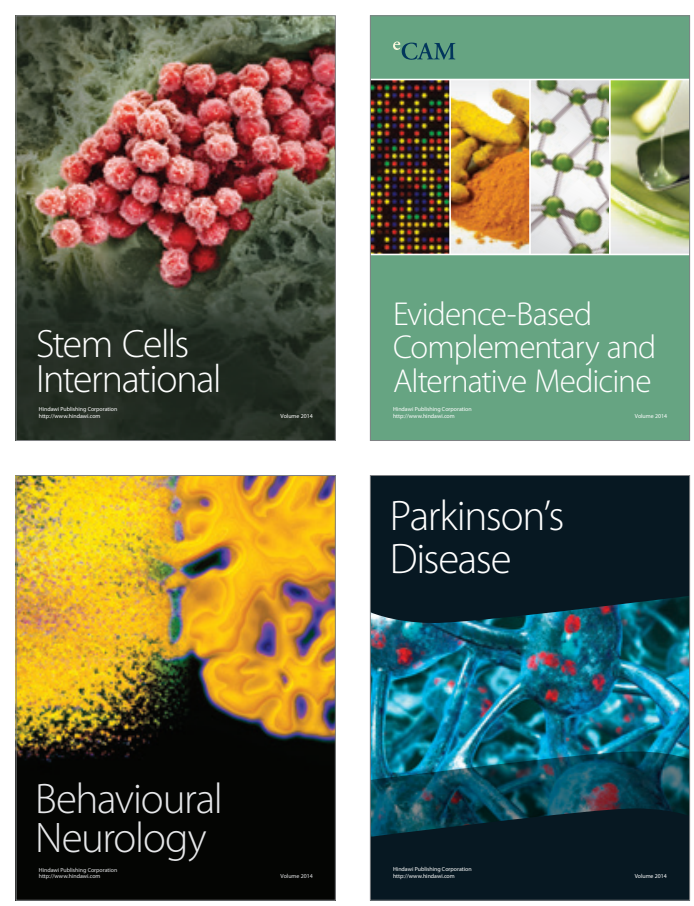

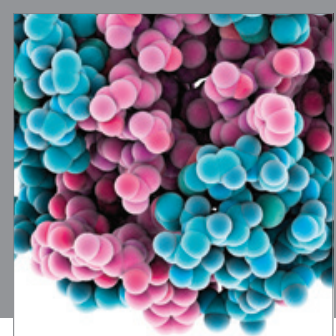

Journal of
Diabetes Research

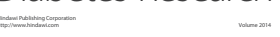

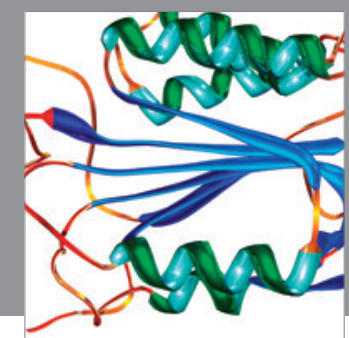

Disease Markers
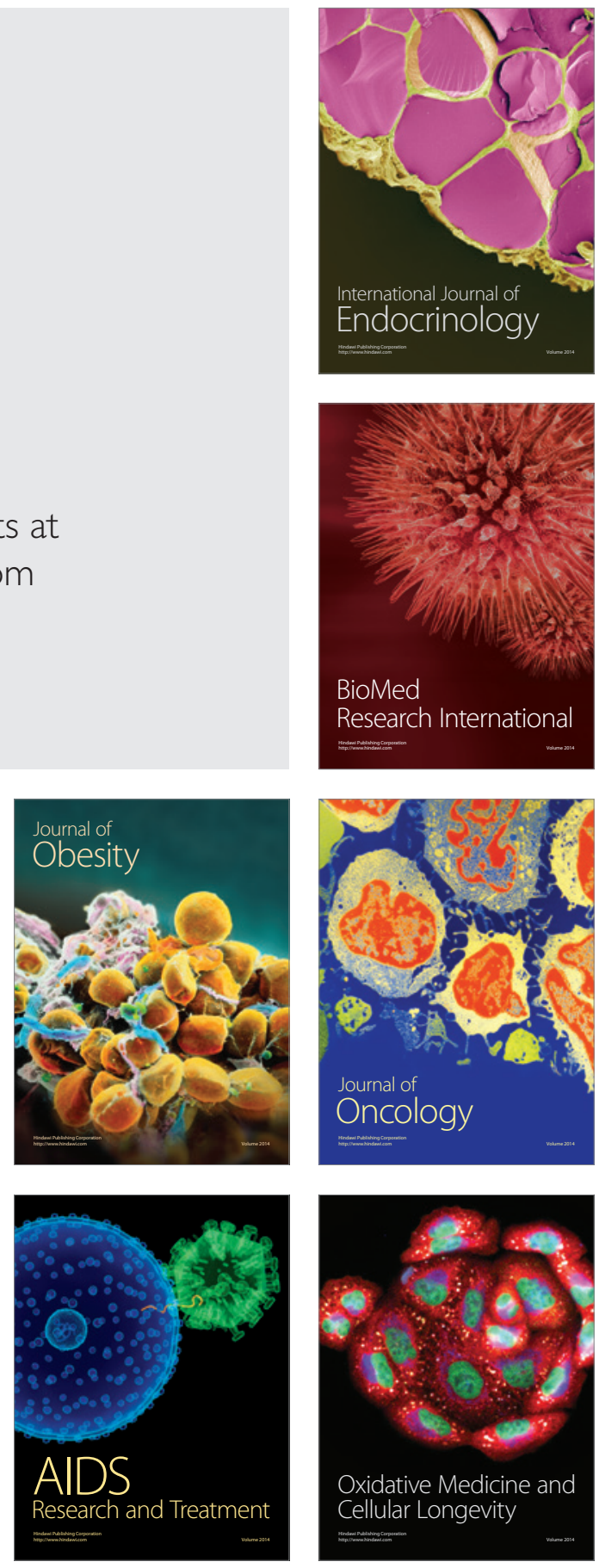